\title{
AVALIAÇÃO DO PERFIL E DA RESPOSTA SEXUAL DE ACADÊMICAS DE UMA INSTITUIÇÃO DE ENSINO SUPERIOR NO CENTRO-NORTE DO PARANÁ
}

\author{
ASSESSMENT OF THE PROFILE AND SEXUAL RESPONSE OF \\ ACADEMICS OF A HIGHER EDUCATION INSTUTION IN CENTRAL NORTH \\ OF PARANÁ
}

Diene Antunes Mendes ${ }^{1}$, Mayline Nadriani Bernardi ${ }^{2}$, Luciana Cristina Rafael Ognibeni $3^{*}$

${ }^{1}$ Graduada em Fisioterapia pelo Centro Universitário Ingá - UNINGÁ (Maringá/PR, Brasil).

${ }^{2}$ Fisioterapeuta especialista em Dermatologia Estética e Uroginecologia Obstetrícia pelo Centro Universitário Ingá - UNINGÁ (Maringá/PR, Brasil).

${ }^{3}$ Mestre em Saúde Coletiva pela UERJ. Fisioterapeuta docente da área de Saúde da Mulher no Centro Universitário Ingá - UNINGÁ (Maringá/PR, Brasil).

${ }^{*}$ Rua Pioneiro Anselmo Cidinei Tait, № 132 - Jardim Fregadolli - Maringá/PR. CEP: 87053-282.

Email: lu.ognibeni@gmail.com

Submetido em: 23/10/2019; Aceito em: 06/05/2020.

\section{RESUMO}

O presente estudo teve como objetivo avaliar o perfil e a resposta sexual de acadêmicas de uma Instituição de Ensino Superior no Centro-Norte do Paraná do curso de Fisioterapia. Foram inclusas 45 acadêmicas sexualmente ativas com idade acima dos 17 anos. Avaliou-se a idade, religião, situação socioeconômica, estado civil, ciclo menstrual, uso de anticoncepção e história sexual e os domínios da sexualidade feminina: desejo, excitação, lubrificação vaginal, orgasmo, satisfação e dor. A idade de início da vida sexual variou de 13 a 23 anos, a maioria era católica, tinham de 2 a 5 salários mínimos, namoravam, tinham ciclo menstrual regular e faziam uso de anticoncepção por via oral. Quanto ao número de parceiros, até hoje, variou de 1 a 16 , no que diz respeito ao número de parceiros atual foi de apenas de 1 e a frequência de coitos 2 vezes por semana. Por meio da aplicação do questionário IFSF, foi possível identificar que houve correlação entre a idade de início sexual e lubrificação; a idade atual e orgasmo; os números de parceiros até hoje teve correlação com desejo, excitação, lubrificação e satisfação; os números de parceiros atuais teve correlação somente com desejo e a frequência de coitos por semana não houve correlação. Pode-se concluir que o questionário IFSF foi um instrumento de fácil aplicabilidade para avaliar a qualidade da resposta sexual das acadêmicas e muito utilizada em pesquisas por poder avaliar cada domínio da resposta sexual e converte medidas subjetivas em dados objetivos e quantificáveis.

Palavras-chave: Ciclo da resposta sexual. Disfunção sexual. Fisioterapia. 


\section{ABSTRACT}

The present had as objective to evaluate the profile and sexual response of academics from a Institution in the Center-North of Paraná for the Physiotherapy course. 45 sexually active academics over the age of 17 were included. Age, religion, socioeconomic status, marital status, menstrual cycle, use of contraception and sexual history and the domains of female sexuality were evaluated: desire, excitement, vaginal lubrication, orgasm, satisfaction and pain. The age of outset of sexual life ranged from 13 to 23 years, most were Catholic, had 2 to 5 minimum wages, dated, had a regular menstrual cycle and used oral contraception. As for the number of partners, to date, it has varied from 1 to 16 , with regard to the number of current partners it was only 1 and the frequency of coitus 2 times a week. Through the application of the IFSF questionnaire, it was possible to identify that there was a correlation between the age of sexual onset and lubrication; current age and orgasm; the number of partners to date has been correlated with desire, excitement, lubrication and satisfaction; the numbers of current partners were correlated only with desire and the frequency of intercourse per week was not correlated. It can be concluded that the IFSF questionnaire was an easily applicable instrument to assess the quality of the sexual response of the students and was widely used in research because it was able to evaluate each domain of the sexual response and converts subjective measures into objective and quantifiable data.

Keywords: Cycle of sexual response. Physiotherapy. Sexual dysfunction.

\section{INTRODUÇÃO}

É cada vez mais validada a relevância da saúde sexual para duração das relações afetivas e como parte da saúde e bem-estar do indivíduo. Atualmente, independente do gênero, o prazer do sexo tem mostrado maior importância do que a própria finalidade reprodutiva (STUDD, 2007).

Nos últimos dez anos, as mulheres têm procurado aos cuidados médicos, com maior frequência, em busca de intervenções para os problemas que interfere na qualidade de vida, principalmente aqueles relacionados com a função sexual. Entretanto, menos de $10 \%$ dos médicos têm a iniciativa de indagar sobre as queixas sexuais de suas pacientes (MARTINEZ, 2008).

Uma grande parcela de mulheres não busca ajuda médica, por vergonha, frustração ou por falhas de tentativas de tratamentos realizados por profissionais não capacitados. Uma minoria das mulheres tem a iniciativa de falar sobre suas dificuldades sexuais e apenas uma pequena parcela dos ginecologistas questiona sobre a função sexual de suas pacientes (BERMAN, et al., 2003).

As alterações da resposta sexual podem interferir tanto na qualidade de vida das mulheres como no relacionamento com os seus parceiros. Pode influenciar a saúde física e mental e pode ser afetada por fatores orgânicos, sociais e econômicos (FERREIRA; SOUZA; AMORIM, 2007). 
A fase inicial da resposta sexual é o desejo, seguida de quatro fases sendo excitação, platô, orgasmo e resolução. A resposta sexual indica que 0 estímulo sexual interno é provocado por pensamentos e fantasias, assim como o externo estimulado por tato, olfato, audição, gustação e visão, promovendo então a excitação, vaso congestão da vagina e da vulva e lubrificação vaginal (ABDO; FLEURY, 2006).

A primeira mudança que ocorre é o aumento no fluxo sanguíneo genital e a contração dos músculos perineais. A lubrificação vaginal inicia-se poucos segundos e com o aumento da lubrificação, a penetração do pênis se torna mais fácil (HALBE, 2000).

A fase de platô é um período relativamente curto e intenso, a tensão sexual e os sentimentos eróticos aumentam e a vaso congestão alcança a intensidade máxima. Após essa fase, ocorre o orgasmo onde os músculos perivaginais e perineais que envolvem 0 terço externo da vagina ficam ingurgitados e contraem-se. Após o orgasmo, as alterações fisiológicas revertem-se e o corpo retorna ao estado de não excitação ou resolução. Depois de meia hora ou mais, o inchaço pélvico diminui e o clitóris, a vagina e o útero voltam à condição normal. Após o orgasmo, as mulheres experimentam uma sensação de relaxamento e bem-estar. A interrupção em uma das fases que determina o ciclo da resposta sexual, é chamada disfunção sexual (BEREK; ADASH; HILLARD, 1998; BERMAN; BERMAN, 2002; FERREIRA; SOUZA, AMORIM, 2007).

A Disfunção Sexual (DS) é a incapacidade de participar da atividade sexual com satisfação. As disfunções acometem ambos os sexos. Contudo, entre as mulheres as queixas relacionadas à qualidade da experiência sexual como um todo, sobrepujam a falha de uma resposta restrita a um aspecto do ato sexual. As Disfunções Sexuais femininas, assim como as masculinas, são representadas pela falta, excesso, desconforto e/ou dor durante o ciclo de resposta sexual, o que afeta uma ou mais das fases desse ciclo, podendo então bloqueá-lo em determinado momento do seu desenrolar. Quanto mais precoce no ciclo o comprometimento, mais prejuízo trará à resposta sexual e mais complexa serão o quadro clínico, prognóstico e tratamento (ABDO, 2004).

No Brasil, foi realizada uma avaliação com 1.219 mulheres, observaram que $49 \%$ tinham ao menos uma disfunção sexual, sendo $26,7 \%$ disfunção do desejo, $23 \%$ dispareunia e $21 \%$ disfunção do orgasmo. A falta de conhecimento e falta de informações sobre a fisiologia da resposta sexual, medicamentos, condições uroginecológicas patológicas, problemas de ordem pessoal e conflitos conjugais pode provocar sérios problemas emocionais nas mulheres e resultar em alguma disfunção sexual (ABDO, 2004; FERREIRA; SOUZA; AMORIM, 2007).

As causas de disfunção sexual nas mulheres são multifatoriais, envolvendo aspectos físicos, psicológicos, sociais ou até mesmo sendo de causa desconhecida. As mais mostradas são a idade acima de 44 anos, o déficit de estrogênio pela menopausa, as cirurgias vaginais, as disfunções sexuais do parceiro, a crença religiosa e uma baixa percepção da qualidade de vida. Além disso, consumo de álcool ou drogas, gravidez, doenças crônicas e o desuso da musculatura perineal também têm sido mencionados como causas de 
disfunção (MASTERS; JOHNSON, 1979; WEBER et al., 1995; PÉREZ; SIGLER; GENOVÉS, 2006).

Quanto ao início da Disfunção Sexual, pode ser classificado em tipos, como: ao longo da vida, que está presente desde o início da vida sexual e o tipo adquirido, desenvolve após um período de desempenho normal (KAPLAN, 1977; TOZO, 2007).

Já, quanto ao contexto em que ocorre, apresenta o tipo generalizado, que não está limitada a estimulação, situação ou parceiros; e o tipo situacional, está limitado a algum tipo de estimulação, situação ou parceiros (KAPLAN, 1977; BERG, 2000; VIDEBECK, 2012).

Durante o Congresso Internacional de Disfunção Sexual Feminina, realizado em Paris, estabeleceu um consenso para classificar as disfunções sexuais femininas em distúrbios do desejo sexual, no qual se encaixa desejo sexual hipoativo e a aversão sexual, os distúrbios da excitação sexual, a anorgasmia e por último os distúrbios de dor durante 0 ato sexual que abrange a dispareunia, vaginismo e também a dor sexual não-coital (KAPLAN, 1977).

Diante do contexto o treinamento dos músculos do assoalho pélvico (TMAP) e a conscientização dos músculos do assoalho pélvico (MAP) têm sido mencionados como técnicas auxiliares no tratamento das disfunções sexuais femininas por alterarem de maneira positiva a vida sexual (KAPLAN, 1974; CHAMBLESS et al., 1984; SHAFIK, 2000). Frente ao exposto acima, esta pesquisa tem como objetivo avaliar o perfil e a resposta sexual das acadêmicas do curso de Fisioterapia.

\section{MATERIAL E MÉTODOS}

Trata-se de um estudo transversal, de caráter descritivo analítico, tendo como base aplicação de questionários em acadêmicas do curso de Fisioterapia noturno de uma Instituição de ensino superior no Centro-Norte do Paraná, a partir da aprovação do Comitê de Ética e Pesquisa em Seres Humanos (CEP UNINGÁ) sob o parecer 2.612.387, em 20/04/2018.

A pesquisa foi composta por 81 acadêmicas acima de 17 anos, que iniciaram a vida sexual há pelo menos um ano. Foram excluídas aquelas que responderam o questionário de forma incorreta ou incompleta. A aplicação dos questionários foi realizada mediante autorização da coordenação do curso de Fisioterapia, para que a pesquisadora entrasse em sala no horário de aula. A pesquisadora inicialmente explicou às acadêmicas o objetivo da pesquisa e aquelas que aceitaram participar assinaram o Termo de Consentimento Livre e Esclarecido.

No segundo momento, as acadêmicas responderam o questionário de anamnese sexual contendo dados sobre a idade, religião, situação sócia econômica, estada civil, ciclo menstrual, usa de anticoncepção e história sexual. Em seguida foi aplicado o questionário IFSF - Índice de Função Sexual Feminina, composto por 19 questões onde se avaliou seis domínios da sexualidade feminina: desejo, excitação, lubrificação vaginal, orgasmo, satisfação e dor. Analisadas dentro das últimas quatro semanas, em que teve relação sexual, conforme apresentado na Tabela 1. 
São questões de múltiplas escolhas e para cada questão existe um padrão de resposta onde às opções recebem pontuação de 0 a 5 de forma crescente em relação à presença da função questionada. Somente nas questões de dor a pontuação é definida de forma invertida.

Tabela 1 - Escore de Avaliação IFSF

\begin{tabular}{lccc}
\hline Domínio & Questões & Variação do Escore & Fator de Multiplicação \\
\hline Desejo & 1,2 & $1-5$ & 0,6 \\
Excitação & $3,4,5,6$ & $0-5$ & 0,3 \\
Lubrificação & $7,8,9,10$ & $0-5$ & 0,3 \\
Orgasmo & $11,12,13$ & $0-5$ & 0,4 \\
Satisfação & $14,15,16$ & $0($ ou 1$)-5$ & 0,4 \\
Dor & $17,18,19$ & $0-5$ & 0,4
\end{tabular}

Fonte: Pacagnella; Martinez; Vieira (2009).

Após os dados coletados, os mesmos foram submetidos à análise estatística.

Para análise foi aplicado teste de normalidade de Shapiro - Wilk. Análises descritivas foram apresentadas: médias e desvio padrão, frequência e porcentagem. Para verificar correlação entre as amostras, a Correlação de Spearman foi utilizada. Os dados foram avaliados com auxílio do programa estatístico SPSS v. 23. Para correlação, valores de $\mathrm{P}<0,05$ indicam correlação entre as variáveis. Valores $>0,05$ não há correlação.

Em relação aos dados de correlação, o $R$ significa uma correlação positiva (quando os dois valores aumentam) ou uma correlação negativa (quando um aumenta e o outro diminui) uma correlação "perfeita" tem o valor de $R=1$.

\section{RESULTADOS E DISCUSSÃO}

Apenas 45 acadêmicas responderam corretamente os questionários e 36 responderam as questões de forma incorreta ou incompleta. A idade de início da vida sexual variou de 13 a 23 anos, com idade média de 16,62 anos.

Segundo Rissel et al. (2003), a proporção de jovens brasileiros que iniciaram a vida sexual, na faixa etária entre 16 e 19 anos e sexualmente ativos foi de $61 \%$ e a idade média do início da vida sexual foi de 14,9 anos.

No que diz respeito ao número de parceiros até hoje, os dados mostraram que variou de 1 a $16 \mathrm{com}$ média de 3,37 parceiros, conforme mostra a Tabela 2.

Tabela 2 - Caracterização sobre a anamnese sexual

\begin{tabular}{lcc}
\hline Anamnese Sexual & Média & Desvio padrão \\
\hline Início da vida sexual & 16,62 & 2,30 \\
Número de parceiros até hoje & 3,37 & 3,31 \\
Número de parceiros atualmente & 1,02 & 0,14 \\
Frequência de coitos/semanal & 2,42 & 1,09 \\
\hline
\end{tabular}

Fonte: os autores. 
De acordo com o Centro Brasileiro de Análise e Planejamento, o histórico de quatro ou mais parceiros sexuais durante toda vida ou dois ou mais parceiros nos últimos dois meses, é considerado fator de risco importante para DST e AIDS (MONTEIRO, 1998).

Neste estudo, verificou que não houve correlação com o autor acima citado, pois quanto ao número de parceiros atuais, $97,8 \%$ das acadêmicas tiveram apenas 1 parceiro. A frequência de coitos foi de duas vezes por semana $(42,2 \%)$ seguida de 3 vezes $(26,7 \%)$. Não foram encontradas pesquisas que discutisse a quantidade de coitos por semana.

O presente estudo mostrou que $73,3 \%$ das entrevistadas são católicas, seguida de 24,4\% evangélicas e apenas 2,2\% protestantes (Tabela 2). Alguns estudos apontam que a religiosidade tende a atrasar o início da vida sexual. As correlações entre religiões e comportamento sexual ainda é pouco estudado na literatura científica e mais estudos são necessários no Brasil (HARDY, 2003).

Através dos dados coletados, quanto à situação socioeconômica $62,2 \%$ apresentaram de 2 a 5 salários mínimos. Madkour et al. (2010), descreve em seu estudo que o nível socioeconômico e escolaridade são fatores que tem relação ao início da atividade sexual precoce. Sendo assim, baixa renda familiar e pouca escolaridade pode ter influência para iniciar uma vida sexual precoce devida à precipitação de algumas fases evolutivas.

De acordo com os achados desse estudo, no que diz respeito ao estado civil, $55,6 \%$ estão em contrato de união estável. O fato de a grande maioria das mulheres ter sua primeira relação sexual com namorado (a), enquanto os homens tiveram a primeira relação sexual com ficante, reforça a suspeita que a atividade sexual para as mulheres relaciona-se com o emocional (BORGES, 2007).

Quanto ao ciclo menstrual, 95,6\% relataram ter um ciclo regular. Apesar de a menarca ser considerada um período marcante para as mulheres, por ser o início da vida reprodutiva e apresentar grandes transformações, alguns autores indagam se é um indicador de maturação adequado, pois, apesar de acontecer em uma determinada idade, uma garota é considerada sexualmente madura quando o ciclo menstrual se tornasse regular (KLUG; FONSECA, 2008).

Quanto à anticoncepção, $71,1 \%$ fazem uso de anticoncepcional por via oral, seguido de $17,8 \%$ de preservativo masculino. Paiva (2000) descreve em seu estudo que as mulheres também amplificaram o uso de anticonceptivos, os resultados mostraram que o preservativo masculino foi parcialmente trocado por outros métodos, como o contraceptivo hormonal oral ou injetável.

Apesar de as mulheres entrevistadas terem iniciado a vida sexual fora de uma união e em idade mais precoce, elas trocaram o preservativo como método contraceptivo de escolha por estarem em um relacionamento mais estável e com vínculo amoroso, comprovando uma tendência de abandono do preservativo masculino na duração do relacionamento, como prova de amor, confiança e fidelidade ao parceiro ou como decorrência de uma sensação de proteção e imunidade ligadas ao amor.

Por meio da aplicação do questionário FSFI, foi possível observar a correlação entre idade de início da vida sexual $x$ lubrificação $(p=0,030)$, idade atual $x$ orgasmo $(p=0,002)$, números de parceiros até hoje $x$ desejo $(p<0,001)$, 
excitação $p(<0,001)$, lubrificação $(p<0,001)$ e satisfação $(p=0,004)$. Pode-se observar correlação entre números de parceiros atuais $x$ desejo $(p=0,004)$ e na frequência de coitos/semanal não houve correlação com nenhum domínio, é importante salientar que o domínio dor não teve correlação com nenhuma das variáveis, conforme mostrado nas Tabelas 3 e 4.

Tabela 3 - Caracterização sócio demográfica das acadêmicas.

\begin{tabular}{lcc}
\hline Religião & N & $\%$ \\
\hline Católica & 33 & 73,3 \\
Congregação Cristã no Brasil & 1 & 2,2 \\
Evangélica & 10 & 22,2 \\
Protestante & 1 & 2,2 \\
Total & 45 & 100,0 \\
\hline Situação Sócio Econômica & 28 & \\
\hline 2 a 5 salários mínimos & 1 & 62,2 \\
6 a 10 salários mínimos & 16 & 2,2 \\
Até 1 salário mínimos & 45 & 35,6 \\
Total & & 100,0 \\
\hline Estado Civil & 10 & 22,2 \\
\hline Casada & 25 & 55,6 \\
União Estável & 10 & 22,2 \\
Solteira & 45 & 100,0 \\
Total & & \\
\hline Ciclo Menstrual & 2 & 4,4 \\
Irregular & 43 & 95,6 \\
Regular & 45 & 100,0 \\
Total & & \\
\hline Anticoncepção & 32 & 71,1 \\
Anticoncepção de via oral & & \\
Anticoncepção de via oral e preservativo & 4 & 8,9 \\
masculino & 1 & 2,2 \\
DIU & 8 & 17,8 \\
Preservativo masculino & 45 & 100,0 \\
Total & & \\
\hline
\end{tabular}

Fonte: os autores.

Sendo assim, as mulheres que iniciaram a vida sexual poderão apresentar dificuldade em atingir um dos domínios, porém, com o desenvolver da prática sexual vão conseguindo atingir o limiar, desta forma, não significa que tenham disfunção sexual caso não atinjam outros domínios. Com o passar do tempo é possível que os domínios sejam atingidos pela experiência sexual adquirida. 
Tabela 4 - Caracterização dos questionários IFSF.

\begin{tabular}{lcccc}
\hline \multirow{2}{*}{ Domínios } & \multicolumn{2}{c}{ Idade de Início sexual } & \multicolumn{2}{c}{ Idade Atual } \\
\cline { 2 - 5 } & $\mathbf{R}$ & $\mathbf{P}$ & $\mathbf{R}$ & $\mathbf{P}$ \\
\hline Desejo & 0,124 & 0,244 & $-0,014$ & 0,897 \\
Excitação & 0,133 & 0,075 & 0,091 & 0,222 \\
Lubrificação & $-0,77$ & 0,030 & 0,305 & 0,692 \\
Orgasmo & 0,196 & 0,008 & 0,076 & 0,002 \\
Satisfação & 0,133 & 0,130 & $-0,44$ & 0,556 \\
Dor & $-0,102$ & 0,240 & $-0,042$ & 0,632 \\
Total & 0,055 & 0,110 & 0,007 & 0,841 \\
\hline
\end{tabular}

Notas: $p>0,05$.

Fonte: os autores.

Tabela 5 - Caracterização dos questionários IFSF

\begin{tabular}{lcccccc} 
& \multicolumn{2}{c}{$\begin{array}{c}\text { Número de } \\
\text { parceiros até hoje }\end{array}$} & \multicolumn{2}{c}{$\begin{array}{c}\text { Número de } \\
\text { parceiros atuais }\end{array}$} & \multicolumn{2}{c}{$\begin{array}{c}\text { Frequência de coito/ } \\
\text { semanal }\end{array}$} \\
\cline { 2 - 7 } Domínios & $\mathbf{R}$ & $\mathbf{P}$ & $\mathbf{R}$ & $\mathbf{P}$ & $\mathbf{R}$ & $\mathbf{P}$ \\
\hline Desejo & 0,388 & $<0,001$ & 0,099 & 0,004 & $-0,003$ & 0,980 \\
Excitação & $-0,347$ & $<0,001$ & $-0,136$ & 0,068 & $-0,10$ & 0,899 \\
Lubrificação & $-0,373$ & $<0,001$ & $-0,007$ & 0,925 & $-0,036$ & 0,634 \\
Orgasmo & 0,058 & 0,503 & $-0,078$ & 0,366 & $-0,074$ & 0,391 \\
Satisfação & $-0,246$ & 0,004 & 0,049 & 0,573 & 0,003 & 0,970 \\
Dor & 0,018 & 0,835 & 0,001 & 0,987 & 0,184 & 0,033 \\
\hline Total & $-0,044$ & 0,196 & $-0,035$ & 0,300 & 0,015 & 0,660 \\
\hline
\end{tabular}

Notas: $p>0,05$.

Fonte: as autoras.

De acordo com os estudos de Leiblum (2000), as mulheres jovens ou iniciantes costumam apresentar dificuldade para relaxamento ou lubrificação, 0 que é bastante compreendido e não significa disfunção, enquanto não houver experiência sexual suficiente. Entretanto, há aquelas que não têm desejo espontâneo, ou ao tê-lo, nem sempre dão sequência ao ato sexual; algumas iniciam o ato para acompanhar seus parceiros, não motivadas por estímulos próprios. O reconhecimento de sensações genitais aprazível acaba por desencadear nelas o interesse por sexo, o que confirma que a excitação pode se antecipar ao desejo.

Basson et al. (2004) indica um modelo circular para o ciclo de resposta sexual da mulher, em que a ausência de desejo sexual (no início do ciclo) não 
significa disfunção sexual, o que exclui muitas mulheres da classificação de disfunção sexual.

De acordo com os achados deste estudo, foi possível observar que houve correlações entre as variáveis e os domínios, sendo que, não houve dificuldade para atingir pelo menos um dos domínios avaliados, com exceção da frequência de coitos semanal.

Quanto ao número de parceiros até hoje devido à experiência sexual, as acadêmicas conseguiram atingir parcialmente os domínios, e mesmo não chegando ao orgasmo, estão satisfeitas com a relação sexual.

Sendo assim, os resultados deste estudo vão de acordo com a literatura quando o autor Leiblum (2000), diz que as mulheres jovens geralmente apresentam dificuldade para manter o ciclo da resposta sexual até o final do ato sexual, portanto, essas dificuldades não significam disfunção sexual.

\section{CONCLUSÃO}

De acordo com os achados na anamnese sexual, foi possível observar que a idade do início da vida sexual variou de 13 a 23 anos, a maioria eram católicas, tinham de 2 a 5 salários mínimos, estão em contrato de união estável, tinham um ciclo menstrual regular e faziam uso de anticoncepção por via oral. Quanto ao número de parceiros até hoje variou de 1 a 16 parceiros, no que diz respeito ao número de parceiros atual foi de apenas 1 e a frequência de coitos foi 2 vezes por semana.

Por meio da aplicação do questionário IFSF, foi possível identificar que houve correlação entre a idade de início sexual e lubrificação; na idade atual e o orgasmo; os números de parceiros até hoje teve correlação com desejo, excitação, lubrificação e satisfação; o número de parceiros atual teve correlação somente com desejo e a frequência de coitos por semana não houve correlação. A dor não teve correlação com nenhuma das variáveis.

Pode-se concluir que o questionário IFSF foi um instrumento de fácil aplicabilidade para avaliar a qualidade da resposta sexual das acadêmicas e muito utilizado em pesquisas por poder avaliar cada domínio da resposta sexual e converte medidas subjetivas em dados objetivos e quantificáveis.

\section{REFERÊNCIAS}

ABDO, C. H. N. Descobrimento sexual do Brasil. São Paulo: Summus, 2004.

ABDO, C. H. N.; FLEURY, H. J. Aspectos diagnósticos e terapêuticos das disfunções sexuais femininas. Revista de Psiquiatria Clínica; v. 33, p. 162-7, 2006.

BASSON, R. et al. Sexual medicine: Sexual Dysfunctions in Men and Women. 4. Ed., Paris: Health Publications, p. 975-985, 2004.

BEREK, J. S.; ADASH, E. Y.; HILLARD, P. A. Tratado de ginecologia. 12. ed. Rio de Janeiro: Guanabara Koogan, p. 205-16, 1998. 
BERG, O. Afinal, o que é disfunção erétil? Verdades e mentiras sobre a impotência sexual. Dunya: Rio de Janeiro, 2000.

BERMAN, J. R.; BERMAN, L. A. Female sexual function and dysfunction. In: 97 th Annual Meeting, Orlando, EUA, p. 1-17, 2002.

BERMAN, L. et al. Seeking help for sexual function complaints: what gynecologists need to know about the female patient's experience. Fertil Steril, v. 79 , n. 3, p. 572-6, 2003.

BORGES, A. L. V. Relações de gênero e iniciação sexual de mulheres adolescentes. Revista da Escola de Enfermagem da USP, v. 41, n. 4, p. 597604, 2007.

CHAMBLESS, D. L. et al. Effect of pubococcygeal exercise on coital orgasm in women. Journal of Consulting and Clinical Psychology, v. 52, n. 1, p. 114-8, 1984.

Centro Brasileiro de Análise e Planejamento - CEBRAP. Comportamento sexual da população brasileira e percepção sobre HIV e AIDS. Brasília: Ministério da Saúde, 2000.

FEREIRRA, A. L. C. G.; SOUZA, A. I.; AMORIM, M. M. R. Prevalência das disfunções sexuais femininas em clínica de planejamento familiar de um hospital escola no Recife, Pernambuco. Revista Brasileira de Saúde Materno Infantil, v. 7, n. 2, p. 143-50, 2007.

HALBE, H. W. Tratado de ginecologia. 3. Ed. São Paulo: Roca, p. 1923-52, 2000.

HARDY, S. A.; RAFFAELLI, M. Adolescent religiosity and sexuality: an investigation of reciprocal influences. Jounal of Adolescence, v. 26, n. 6, p. 731-9, 2003.

KAPLAN, H. S. A nova terapia do sexo. 4. ed. Rio de Janeiro: Nova Fronteira, 1974.

KAPLAN, H. S. A nova terapia do sexo. 3. ed. Rio de Janeiro: Nova Fronteira, p. $493,1977$.

LEIBLUM, S. R. Redefining female sexual response. Contemporary Obstetrics \& Gynecology, v. 45, p. 120-126, 2000.

KLUG, D. P.; FONSECA, P. H. S. Análise da maturação feminina: um enfoque na idade de ocorrência da menarca. Revista da Educação Física/UEM, v. 17, n. 2, p. 139-47, 2008. 
MADKOUR, A. S. et al. Early adolescent sexual initiation as a problem behavior: a comparative study of five nations. Journal of Adolescent Health, v. 47, p. 389-98, 2010.

MARTINEZ, L. More education in the diagnosis and management of sexual dysfunction is needed. Fertility and Sterility, v. 89, n. 4, p. 1035, 2008.

MASTERS, W. H.; JONHSON, V. E. A conduta sexual humana 3. ed. Rio de Janeiro: Civilização Brasileira, 1979.

MONTEIRO, L. C. Conhecimentos e crenças sobre doenças sexualmente transmissíveis/AIDS e comportamento sexual em jovens de escolas públicas de Goiânia. [Dissertação]. Goiânia: Universidade Federal de Goiás. Instituto de Patologia Tropical e Saúde Pública, 1998.

PACAGNELLA, R. C.; MARTINEZ, E. Z.; VIEIRA, E. M. Validade de construto de uma versão em português do Female Sexual Function Index. Cadernos de Saúde Pública, Rio de Janeiro, v. 25, n.11, p. 2333-2344, 2009.

PAIVA, V. Fazendo arte com camisinha. Sexualidades em tempos de Aids. São Paulo: Summus, 2000.

PÉREZ, V. A.; SIGLER, M. D. G.; GENOVÉS, J. S. Female sexual function and related factors. Atención Primaria, v. 38, n. 6, p. 339-44, 2006.

RISSEL, C. E. et.al. Sex in Australia: first experiences of vaginal intercourse and oral sex among a representative sample of adults. Australian and New Zealand Journal Public Health, v. 27, n. 2, p. 131-7, 2003.

STUDD, J. A comparison of 19th century and current attitudes to female sexuality. Gynecological Endocrinology, v. 23, n. 12, p. 673-81, 2007.

SHAFIK, A. The role of the levator ani muscle in evacuation, sexual performance, and pelvic floor disorders. International Urogynecology Journal and Pelvic Floor Dysfunction, v. 11, n. 6, p. 361-76, 2000.

TOZO, I. M. et al. Disfunção sexual feminina: a importância do conhecimento e do diagnóstico pelo ginecologista. Arquivos Médicos dos Hospitais e da Faculdade de Ciências Médicas da Santa Casa de São Paulo, v. 3, n. 52, p. 94-9, 2007.

VIDEBECK, S. L. Enfermagem em saúde mental e psiquiatria. 5. ed. Porto Alegre: ARTMED, 2012.

WEBER, A. M. et al. Vaginal anatomy and sexual function. Obstetrics \& Gynecology, v. 86, n. 6, p. 946-9, 1995. 